\title{
INTESTINAL ABSORPTION AND PRESYSTEMIC DISPOSITION OF SILDENAFIL CITRATE IN THE RABBIT: EVIDENCE FOR SITE- DEPENDENT ABSORPTIVE CLEARANCE
}

\author{
M. A. Osman* ${ }^{1}$, G. M. El Maghraby ${ }^{1}$ and M. A. Hedaya ${ }^{2}$ \\ ${ }^{1}$ Department of Pharmaceutical Technology and ${ }^{2}$ Department of Clinical Pharmacy, College of \\ Pharmacy,University of Tanta, Tanta, Egypt
}

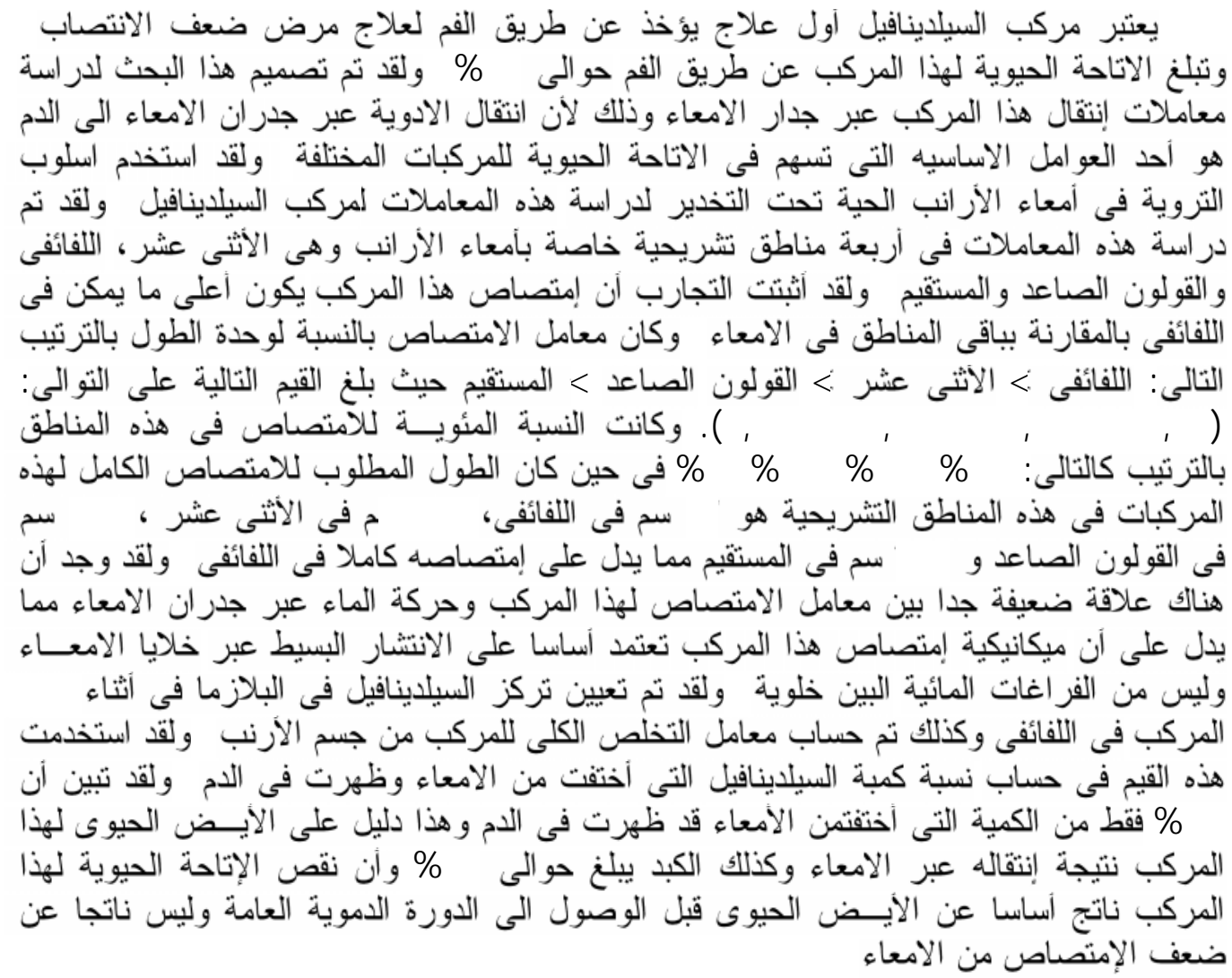

Sildenafil citrate is the first oral treatment of erectile dysfunction. Its oral bioavailability is about $40 \%$. This research characterized the intestinal transport parameters of sildenafil citrate in rabbit using in situ intestinal perfusion technique. This was studied in four different anatomical sites, namely duodenum, jejunoileum, ascending-colon, and rectum. The results revealed the highest absorptive clearance in the jejunoileum. The values of permeability area product normalized to segment length $(\mathrm{ml} / \mathrm{min} \mathrm{cm})$ were $0.0101,0.0063,0.0059$ and 0.0023 and those of percentage absorbed were 67.95, 32.27, 23 and 5.03 in jejunoileum, duodenum, ascending colon, and rectum respectively. The values of the length $(\mathrm{cm})$ required for complete absorption were 87.58, 137.23, 153.27, and 384.09 for each segment in the same order. The absorptive clearance did not correlate with the net water flux in the four anatomical regions studied, indicating mainly passive diffusion mechanism through transcellular pathway. The plasma sildenafil concentrations achieved during intestinal perfusion experiments and sildenafil total body clearance in rabbit were used to calculate the fraction of sildenafil that reached the systemic circulation relative to the amount disappeared from the intestinal segment. Only $34 \%$ of sildenafil disappeared from the intestinal segment appeared in the systemic circulation indicating that the presystemic elimination of slidenafil is $66 \%$. These results confirm that the incomplete bioavailability of sildenafil is mainly due presystemic elimination.

Received in 27/1/2005 \& Accepted in 5/9/2005 


\section{INTRODUCTION}

Sildenafil citrate is the first approved phosphodiestrase-(PDE)-5-selective inhibitor. Its inhibitory effect on the PDE5 is tenfold higher than that on the PDE6, which produces visual changes through the retinal rods, which makes it the drug of choice for the treatment of erectile dysfunction. It has been confirmed that sildenafil citrate is effective therapeutic agent for erectile dysfunction regardless of the etiology. ${ }^{1,2}$ The oral absorption of sildenafil citrate is rapid when administered on an empty stomach, while food slows its absorption leading to delayed onset of action. In addition, rapid onset was observed after sublingual administration when compared with oral administration. The absolute bioavailability of sildenafil after oral administration is only 38$41 \% .^{3-5}$ This incomplete bioavailability could be due to poor absorption from the gastrointestinal tract (GIT), improper targeting to the proper site of absorption in the GIT or due to presystemic elimination of sildenafil. Optimization of drug bioavailability in such case requires identification of the cause of incomplete bioavailability.

The intestinal absorption of xenobiotics could be examined at different levels of integration, in whole animal in vivo, in isolated intestinal segments in situ and in intestinal loops or even enterocytes in vitro. ${ }^{6}$ The in situ methods bypasses the effect of food, drug dissolution and stomach emptying steps after oral dosing while affording input control and choice of the perfused intestinal segment. The in situ methods provide intact lymph, blood and nerve supply for the solute uptake with extended tissue viability demonstrating superiority over in vitro methods. One of these techniques "through-and-through" intestinal perfusion has been employed to monitor the absorption of several compounds, using rabbit as the model animal. ${ }^{7-9}$

The primary objective of this research was to investigate the transport parameters of sildenafil citrate at the level of intestinal membrane in the rabbit. This included qualitative and quantitative investigation of the transport mechanisms of sildenafil citrate across the intestinal membrane, which could be of significant importance in dosage form design and in optimizing the bioavailability of such drug. The membrane transport was studied at four different anatomical sites in the rabbit intestine, namely the duodenum, the jejunoileum, the ascending colon, and the rectum utilizing the through-and-through intestinal perfusion technique. In addition, the presystemic disposition of the drug was also calculated by measuring the concentration of sildenafil appearing in the plasma during the intestinal perfusion experiment. The rabbit was selected in this study as the model animal because the rabbit GIT anatomy and physiology is similar to that of human. ${ }^{10-12}$

\section{EXPERIMENTAL}

\section{Chemicals}

Sildenafil citrate was kindly donated from the Medical Union Professions company (MUP), Ismailia, Egypt, dexametasone was obtained from Sigma chemical Co. (St.Louis, MO, USA), monobasic sodium phosphate from Merck Chemical Co. (Darmstadt, Germany), acetonitrile HPLC - grade from BDH Laboratory (Poole, England), dietyl ether from Honil Limited (London, UK), sodium chloride $0.9 \%$ for injection USP, from El-Nasr Pharmaceutical Chemicals company (Cairo, Egypt). Ketamine $\mathrm{HCl}(100 \mathrm{mg} / \mathrm{ml})$ from EIPICO Pharmaceuticals company (Egypt), and chlorpromazine $\mathrm{HCl}(25 \mathrm{mg} / \mathrm{ml})$ from Misr Pharmaceuticals Company (Egypt).

\section{Isolated intestinal segments preparation}

The procedures for the preparation of the isolated intestinal segments for the in situ perfusion experiment were described in details previously. ${ }^{7}$ Male albino rabbits weighing 2.8$3.1 \mathrm{~kg}$ were used for this procedure. After an overnight fasting, the rabbits were anesthetized by intramuscular (IM) injections of ketamine $\mathrm{HCl}$ (two doses of $45 \mathrm{mg} / \mathrm{kg}$ at 15 minutes interval and additional dose of $25 \mathrm{mg} / \mathrm{kg}$ can be given if needed). Chloropromazine $\mathrm{HCl}$ was used as muscle relaxant and was given before each anesthetic dose. The dose of muscle relaxant was $2 \mathrm{mg} / \mathrm{kg}$ given I.M. Ketamine $\mathrm{HCl}$ was selected as it is the most widely used anesthetic that does not affect the microcirculation of the GIT. ${ }^{7}$

After induction of anesthesia, the rabbit was laid in a supine position on a heating pad and the abdominal area was shaved and cleaned 
with a depilatory cream. A longitudinal incision 6-8 $\mathrm{cm}$ was made, and the intestinal segments of interest were exposed and isolated carefully. In order to cannulate the jejunoileum segment, the proximal end $(60 \mathrm{~cm}$ from the pylorus) was tied off using surgical thread, and was cannulated using a 3-way stopcock cannula. The desired length of the intestinal segment was then measured and the distal end was cannulated using an L-shaped glass cannula. A length of $30 \mathrm{~cm}$ was used throughout the study except in the group which was used to correlate intestinal absorption with plasma concentration where $60 \mathrm{~cm}$ segment was used. The use of longer segment was to ensure detectable concentrations in the plasma.

For the colon, the proximal end was tied off immediately after the ampulla coli, the desired length $(15 \mathrm{~cm})$ was measured, and finally the distal end was tied off. Two incisions were made, one on each end, and the solid fecal debris were squeezed out by gentle manipulation of the segment, and by infusing normal saline through the proximal end. Finally, both the proximal and distal ends were cannulated as described before. The same procedures were applied to both the duodenum and rectum taking the length of the duodenum as $15 \mathrm{~cm}$ and that of the rectum as $5 \mathrm{~cm}$. Each rabbit was utilized to study the absorption from two different intestinal segments in the same time.

The intestinal segment under study was carefully arranged in a uniform S- to multi-Spattern, to avoid kinks and ensure uniformity in the intestinal fluid flow during perfusion. The isolated segment was kept warm and moist by frequent application of $37^{\circ}$ normal saline to a gauze pad covering the intestine. The remainder of the intestine was returned into the abdominal cavity for better maintenance of the temperature. At the end of the experiment the animal was euthanized by injecting an overdose of sodium pentobarbital through the marginal ear vein. The intestinal segments under study were excised and were accurately measured.

\section{Anatomical difference in sildenafil absorption}

The first objective of this research was to characterize the anatomical difference in the absorption of sildenafil citrate from four different anatomical sites of the rabbit GIT; duodenum, jejunoileum, colon, and rectum. For this purpose, six animals divided into two groups $(n=3)$ were used. The first group was utilized to study the absorption from the duodenum and the rectum, while the second group was used to study the absorption from the jejunoileum and the ascending colon.

The perfusion solution $(13.6 \mu \mathrm{g} / \mathrm{ml}$ sildenafil citrate) was prepared in $0.9 \%$ sodium chloride for injection, USP. After surgical preparation of the rabbits, sildenafil citrate solution was perfused into the intestinal segment under investigation at a flow rate of $0.27 \mathrm{ml} / \mathrm{min}$ using constant rate perfusion pump (Harvard-22 Apparatus, Millis, MA, USA). The effluent from the perfused segment was collected at 10-minutes intervals for 120 minutes in 10-ml pre-weighed stoppered tubes. These tubes were weighed again after sample collection, and the effluent weight was recorded as the difference.

The net water flux which result from the transmucosal fluid movement, was determined gravimetrically from the difference of the volume of the intestinal perfusion rate and the volume of the intestinal segment outflow taking the density of the aqueous samples as unity. The concentration of the drug in the effluent was corrected accordingly.

\section{Presystemic disposition of sildenafil absorption}

The second objective of this research was to estimate the presystemic disposition of sildenafil. For this purpose, two groups of rabbits ( $n=3$ each) were used. The rabbits of the first group were prepared as described above for perfusion of a $60 \mathrm{~cm}$ segment of the jejunoileum. The jejunoileum segment was selected in this experiment because the regional absorption studies showed that this segment has the highest sildenafil absorption. Also, the marginal ear vein was cannulated using an ivplacement catheter (Charter Med. Inc., NJ, USA). Blood samples $(1 \mathrm{ml})$ were collected every 10 minutes during the intestinal perfusion and throughout the experiment. Plasma was separated by centrifugation and was frozen at $-20^{\circ}$ until analysis.

The steady state sildenafil concentration achieved during the jejunoileum perfusion is dependent on the total body clearance of sildenafil in the rabbit and the amount of 
sildenafil that reaches the systemic circulation during the jejunoileum perfusion. So, it was necessary to estimate the total body clearance of sildenafil in the rabbits in order to calculate the fraction of the absorbed sildenafil that reached to the systemic circulation. For this purpose, a second group of rabbits was used to calculate the total body clearance of sildenafil. The rabbits were anesthetized as described above and both marginal ear veins were cannulated with two iv catheters. One catheter was used to infuse $110 \mu \mathrm{g} / \mathrm{ml}$ sildenafil at a rate of $0.1 \mathrm{ml} / \mathrm{min}$ after an iv bolus dose of 1 $\mathrm{mg}$ sildenafil, and the second catheter was used to collect blood samples. Blood samples $(1 \mathrm{ml})$ were collected every 30 minutes for a period of 5 hours. Plasma was separated by centrifugation and was frozen at $-20^{\circ}$ until analysis.

\section{Sample Analysis}

Samples were analyzed by HPLC. This analysis employed a high pressure liquid chromatograph (Waters ${ }^{\mathrm{TM}} 600$ controller, Waters, USA) equipped with a variable wavelength detector (Waters ${ }^{\mathrm{TM}}$ 486) and an automatic sampling system (Waters ${ }^{\mathrm{TM}}$ 717). The mobile phase consisted of $20 \mathrm{mM}$ monobasic sodium phosphate/acetonitril (65:35), and the flow rate was $1.3 \mathrm{ml} / \mathrm{min}$. Separation was achieved using a $15 \mathrm{~cm} \mathrm{X} 3.9$ $\mathrm{mm}$ (i.d.) $\mathrm{C}_{18}, \mu$ Bondapak $^{\mathrm{TM}}$, Waters, reversed phase column with an average particle size of $10 \mu \mathrm{m}$, and the column was kept at ambient temperature. The column effluent was monitored at $240 \mathrm{~nm}$ and the chromatographic data analysis was performed with the Millinium ${ }^{\mathrm{TM}}$ Program (Waters, USA).

The perfusate samples collected during the intestinal perfusion were centrifuged for 5 minutes in order to precipitate any mucus debris. An aliquot of $0.5 \mathrm{ml}$ from each perfusate sample, and $0.5 \mathrm{ml}$ of the mobile phase were transferred to a clean test tube spiked with dexamethasone. The final dexamethasone concentration was $2 \mu \mathrm{g} / \mathrm{ml}$. Thirty microliters of the resulting solution was injected into the HPLC. Standard sildenafil solutions were prepared by addition of $1 \mathrm{ml}$ mobile phase to a series of test tubes spiked with the internal standard $(2 \mu \mathrm{g} / \mathrm{ml})$ and sildenafil citrate sufficient to produce a concentration range of $0.1-20 \mu \mathrm{g} / \mathrm{ml}$.
Rabbit plasma samples were analyzed by transferring $0.5 \mathrm{ml}$ of rabbit plasma to clean test tubes spiked with the internal standard to produce dexamethasone concentration of 2 $\mu \mathrm{g} / \mathrm{ml}$. The resulting solution was extracted with $4 \mathrm{ml}$ of diethyl ether for $5 \mathrm{~min}$, and then centrifuged for 5 minutes. The ether layer was transferred to clean test tubes and was evaporated. The residue was reconstituted in $250 \mu \mathrm{l}$ of the mobile phase and $30 \mu \mathrm{l}$ of the resulting solution was injected onto the HPLC. Standard sildenafil samples were prepared similarly by extracting plasma samples spiked with known sildenafil concentration in the range of $0.05-5 \mu \mathrm{g} / \mathrm{ml}$.

\section{Data analysis \\ Absorptive clearance}

The flow rate was estimated for each perfusion experiment from the linear regression of the volume remaining in the perfusion syringe versus time. The volume of the outflow samples was estimated gravimetrically taking the density of the aqueous samples as 1 (the same as water). From the difference in flow rate entering and leaving the intestinal segment, the outflow concentration was corrected for the net water flux. The ratio between the corrected concentration at the outflow $\left\{\mathrm{C}_{(\mathrm{out})}\right\}$ and that at the inflow $\left\{\mathrm{C}_{(\mathrm{in})}\right\}$ was calculated for each perfusate sample collected. The average of the outflow-to-inflow concentration ratios for the fractions collected from 70 to $120 \mathrm{~min}$ was taken as the steady-state ratio. This ratio at steady-state is given by: ${ }^{13-17}$

$$
\left\{\mathrm{C}_{\text {(out) }} / \mathrm{C}_{\text {(in) }}\right\}_{\mathrm{ss}}=\exp ^{-(\mathrm{PeA} / \mathrm{Q})}
$$

Where $\mathrm{A}$ is the effective surface area $\left(\mathrm{cm}^{2}\right), \quad \mathrm{Pe}$ is the apparent permeability coefficient $(\mathrm{cm} / \mathrm{min})$, and Q is the average flow rate within the intestinal segment $(\mathrm{ml} / \mathrm{min})$. Rearrangement of equation (1) allows the permeability-area product $(\mathrm{PeA})$ to be calculated:

$\mathrm{PeA}=-\mathrm{Q} \cdot \ln \left(\mathrm{C}_{\text {(out) }} / \mathrm{C}_{(\text {in })}\right)_{\mathrm{ss}}$

Employing in situ intestinal perfusion technique, the term (PeA) should be normalized to the length of the intestinal segment in order to allow for comparison of the effective permeability of segments having different lengths. 
Since $\left\{\mathrm{C}_{(\text {out })} /\left(\mathrm{C}_{(\text {in })}\right)\right\}_{\mathrm{ss}}$ is the fraction remaining after solution has passed through the intestinal length, then the fraction absorbed is:

$\mathrm{Fa}=1-\left\{\left(\mathrm{C}_{(\text {out })}\right) /\left(\mathrm{C}_{(\text {in })}\right)\right\}_{\mathrm{ss}}=1-\exp ^{-(\mathrm{PeA} / \mathrm{Q})}$

Associated with the concept of intestinal absorption is the reserve length, ${ }^{15}$ the anatomical reserve length (ARL), is defined as the length of the intestine remaining after absorption has been completed, and it is given by:

$\mathrm{ARL}=\left(\mathrm{L}^{*}\right)-\left(\mathrm{l}^{*}\right)$

Where, ARL is the anatomical reserve length $(\mathrm{cm}) . \mathrm{L}^{*}$ is the maximal intestinal length available for absorption. $1^{*}$ is the intestinal length along which absorption is complete, (cm). In theory, the bulk luminal concentration will never be reduced to zero at the intestinal length $\left(1^{*}\right)$, due to the nature of the logarithmic function. Accordingly, an arbitrary small fraction of solute remaining will be considered as the criteria for complete absorption. Taking this fraction as 5\%, and replacing in equation (1) will give the following equation:

$0.05=\exp ^{-\left\{\left(\text {PeA. } 1^{*}\right) / Q\right\}}$

Where, PeA is the effective permeability surface area product normalized to length. $1^{*}$ is the length required for 95\% absorption (L95\% ab.) of a given solute.

\section{Effect of Solvent Drag on Intestinal Absorptive Clearance}

The net amount of drug absorbed per unit time can be described as the sum of two terms; the diffusive contribution and the convective contribution corresponding to the solvent drag effect. The net amount of drug absorbed per unit time is then given by the following equation. $^{18-20}$

$\mathrm{Js}=\mathrm{Ks}\left(\mathrm{C}-\mathrm{C}_{\mathrm{p}}\right)+\varnothing \mathrm{s} \mathrm{J}_{\mathrm{w}} \mathrm{C}$

In which the first term on the right is diffusive and the second is convective and Js is the rate of absorption of the solute from the lumen $(\mu \mathrm{g} / \mathrm{min})$ and is given by

$\Delta \mathbf{N s} / \Delta \mathrm{t}$ where, $\Delta \mathbf{N s}$ is the amount of the solute $(\mu \mathrm{g})$ absorbed in a time interval $\Delta \mathbf{t}(\mathrm{min}) . \mathbf{K s}$ is the diffusive permeability coefficient and is given by DAKp/ $\Delta \mathbf{x}$, in which $\mathbf{D}$ is the diffusion coefficient of the solute, $\mathbf{A}$ is the effective surface area, $\mathbf{K p}$ is the partition coefficient of the compound, and $\Delta \mathbf{x}$ is the path length. $\mathbf{C}$ and $\mathbf{C}_{\mathbf{p}}$ are the solute concentrations in the lumen and plasma, respectively. $\varnothing \mathbf{s}$, is the sieving coefficient of the given compound, represents the ratio between the concentration of the compound in the convective stream to that in the luminal fluid, $\varnothing$ s equals $\mathbf{1}-\sigma$, where $\sigma$, is Staverman reflection coefficient of a given compound, represents its interaction with water. $\mathbf{J w}$ is the rate of fluid (or water) flux without reference to its mechanism (osmotic, hydrostatic or electrical). The water flux is absorption-secretion process which is dependent on the experimental parameters during the perfusion. Jw, Ks, and $\varnothing \mathbf{s}$ are assumed to remain constant during a given experiment run. At the steady state, due to sink conditions in the blood, equation (6) is reduced to

$\mathrm{J}_{\mathrm{ss}}=\mathrm{DAKp} / \Delta \mathrm{x}\left(\mathrm{C}_{\mathrm{ss}}\right)+\varnothing \mathrm{Jw}\left(\mathrm{C}_{\mathrm{ss}}\right)$

where, $\mathbf{J}_{\mathbf{s s}}$ is the steady state solute flux $(\mu \mathrm{g} / \mathrm{min})$ and $\mathbf{C}_{\mathrm{ss}}$ is the length averaged steady state concentration of the solute in the lumen $(\mu \mathrm{g} / \mathrm{ml})$

Rearrangement of equation (7) gives the following equation.

$\mathrm{J}_{\mathrm{ss}} / \mathrm{C}_{\mathrm{ss}}=\mathrm{DAKp} / \Delta \mathrm{x}+\varnothing \mathrm{JW}$

The term $\mathbf{J}_{\mathrm{ss}} / \mathbf{C}_{\mathrm{ss}}$ represents the overall absorptive clearance of the given solute $(\mathrm{ml} / \mathrm{min})$, regardless its route or mechanism, and practically it is estimated as the overall absorptive clearance, (PeA), (equation 2 ).

\section{Calculation of the presystemic elimination of sildenafil}

The steady state plasma concentration achieved during the intestinal perfusion experiment can be expressed by the following term,

$\mathrm{Cp}_{\mathrm{ss}}=\frac{\mathrm{FR}}{\mathrm{CL}_{\text {tot }}}$

where Cpss is the steady state plasma concentration, $\mathrm{F}$ is the fraction of the drug disappeared during the intestinal perfusion that reaches the systemic circulation, $\mathrm{R}$ is the amount of the drug disappeared during the intestinal perfusion and is equal to $\left(R_{\text {in }}-R_{\text {out }}\right)$, and $\mathrm{CL}_{\text {tot }}$ is the total body clearance of the drug. $\mathrm{CL}_{\text {tot }}$ is calculated during the iv infusion of the drug from the infusion rate $\left(\mathrm{K}_{\mathrm{o}}\right)$, and the steady state plasma concentration during the infusion $\left(\mathrm{Cp}_{\mathrm{ss}}\right)$ : 
$\mathrm{Cp}_{\mathrm{ss}}=\frac{\mathrm{K}_{\mathrm{o}}}{\mathrm{CL}_{\mathrm{tot}}}$

F can be calculated from equation (9) by substituting for the calculated values for $\mathrm{R}$, $\mathrm{C}_{\text {Ltot}}$, and plasma sildenafil concentration achieved during the intestinal perfusion experiment.

\section{RESULTS AND DISCUSSION}

The extent of sildenafil absorption was different in the different intestinal segments investigated in this study. The fraction of sildenafil absorbed during the perfusion of different anatomical segments of the rabbit intestine is presented in Figure 1. The absorptive clearance of sildenafil citrate normalized to the intestinal length $(\mathrm{PeA} / \mathrm{L})$ calculated for the different anatomical regions (Table 1) showed significant differences among the studied segments. The absorption of sildenafil was in the order of jejunoileum > duodenum $>$ ascending colon $>$ rectum. This is also revealed from Figure 1. This indicates difference in sildenafil permeability in the different intestinal regions. The calculated values for the mean \pm standard error for absorptive clearance normalized for the intestinal length $(\mathrm{PeA} / \mathrm{L})$ were $0.0101 \pm 0.0028$, $0.0063 \pm 0.0006,0.0059 \pm 0.0006$, and 0.0023 $\pm 0.0003 \mathrm{ml} / \mathrm{min} . \mathrm{cm}$ for the jejunoileum, duodenum, ascending colon, and rectum, respectively. The length required for complete absorption of sildenafil in the different intestinal segment (L95\%) and the anatomical reserve length were calculated and presented in Table 1.

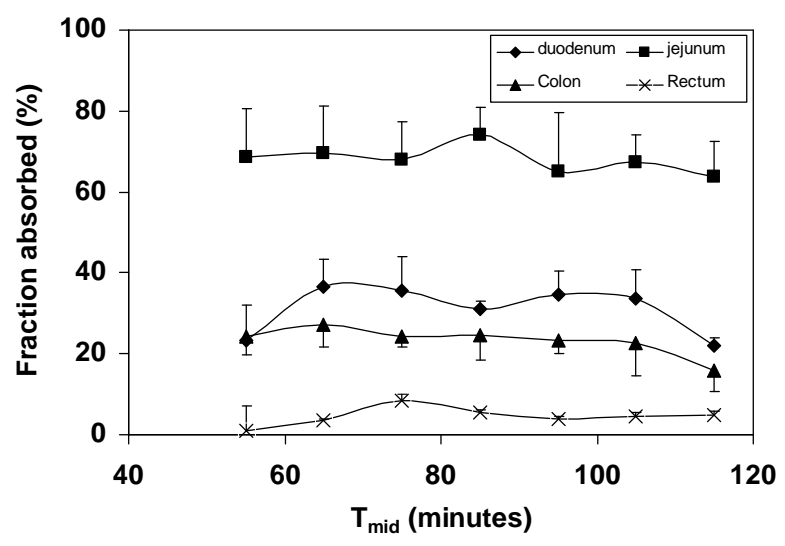

Fig. 1: The fraction of sildenafil absorbed during the perfusion of different anatomical segments of the rabbit intestine. (Data at steady state are presented as mean \pm SEM, $\mathrm{n}=3$ ).

Table 1: Membrane transport parameters of sildenafil in the four different GIT segments.

\begin{tabular}{|c|c|c|c|c|}
\hline Parameter & Duodenum & Jejunoileum & $\begin{array}{c}\text { Ascending } \\
\text { colon }\end{array}$ & Rectum \\
\hline $\begin{array}{c}\text { PeA } \\
(\mathrm{ml} / \mathrm{min})\end{array}$ & 0.104 & 0.321 & 0.067 & 0.013 \\
$(0.0138)$ & $(0.0917)$ & $(0.0083)$ & $(0.0019)$ \\
\hline \multirow{2}{*}{ Rout/Rin } & 0.677 & 0.320 & 0.770 & 0.95 \\
& $(0.03)$ & $(0.09)$ & $(0.03)$ & $(0.01)$ \\
\hline$\%$ Fa & 32.27 & 67.95 & 23.0 & 5.03 \\
& $(3.25)$ & $(9.28)$ & $(2.54)$ & $(0.68)$ \\
\hline $\mathrm{PeA} / \mathrm{L}$ & 0.00633 & 0.0101 & 0.0059 & 0.002323 \\
$(\mathrm{ml} / \mathrm{min} . \mathrm{cm})$ & $(0.0006)$ & $(0.0028)$ & $(0.0006)$ & $(0.0003)$ \\
\hline $1^{*}(\mathrm{~L} 95 \%)$ & 137.2 & 87.58 & 153.27 & 384.1 \\
$(\mathrm{~cm})$ & $(10.1)$ & $(19.97)$ & $(18.75)$ & $(41.44)$ \\
\hline $\mathrm{JW}$ & 0.0296 & 0.0346 & 0.0387 & 0.0332 \\
$(\mathrm{ml} / \mathrm{min})$ & $(0.0014)$ & $(0.0032)$ & $(0.0057)$ & $(0.0129)$ \\
\hline JW/L & 0.0018 & 0.0011 & 0.0034 & 0.0057 \\
$(\mathrm{ml} / \mathrm{min} . \mathrm{cm})$ & $(0.0001)$ & $(0.0002)$ & $(0.0004)$ & $(0.0020)$ \\
\hline \hline
\end{tabular}

Where PeA is the overall absorptive clearance, Rout/Rin is the fraction remaining to be absorbed, \%Fa is the percentage fraction absorbed, $\mathrm{PeA} / \mathrm{L}$ is the effective permeability surface area product normalized to the segment length, L95\% is the length required for $95 \%$ absorption, JW is the water flux and JW/L is the water flux normalized to the segment length. Values between brackets are the standard error, $\mathrm{n}=3$. 
The effect of net water flux on the absorptive clearance of sildenafil was studied according to Lifson's model. ${ }^{18}$ Figure 2 represents a plot of sildenafil absorptive clearance versus the net water flux both normalized to the segment length at steady state for the different intestinal segments. The regression parameters for the different intestinal segments are presented in Table 2. The slopes of the plot for the different segments were not significantly different from zero indicating the absence of paracellular contribution. While the intercepts of the plots were significantly different from zero suggesting mainly transcellular absorption.
The average plasma sildenafil concentration achieved after 6 hours of sildenafil constant rate infusion of $11 \mu \mathrm{g} / \mathrm{min}$ was $0.626 \pm 0.21 \mu \mathrm{g} / \mathrm{ml}$. The differences in the last three sildenafil concentrations were less than $10 \%$ indicating that steady state was achieved. The average total body clearance calculated from sildenafil infusion rate and the average steady state concentration was 17.57 $\mathrm{ml} / \mathrm{min}$. This average total body clearance was used for the calculation of the presystemic elimination parameters for sildenafil during the intestinal perfusion experiment.
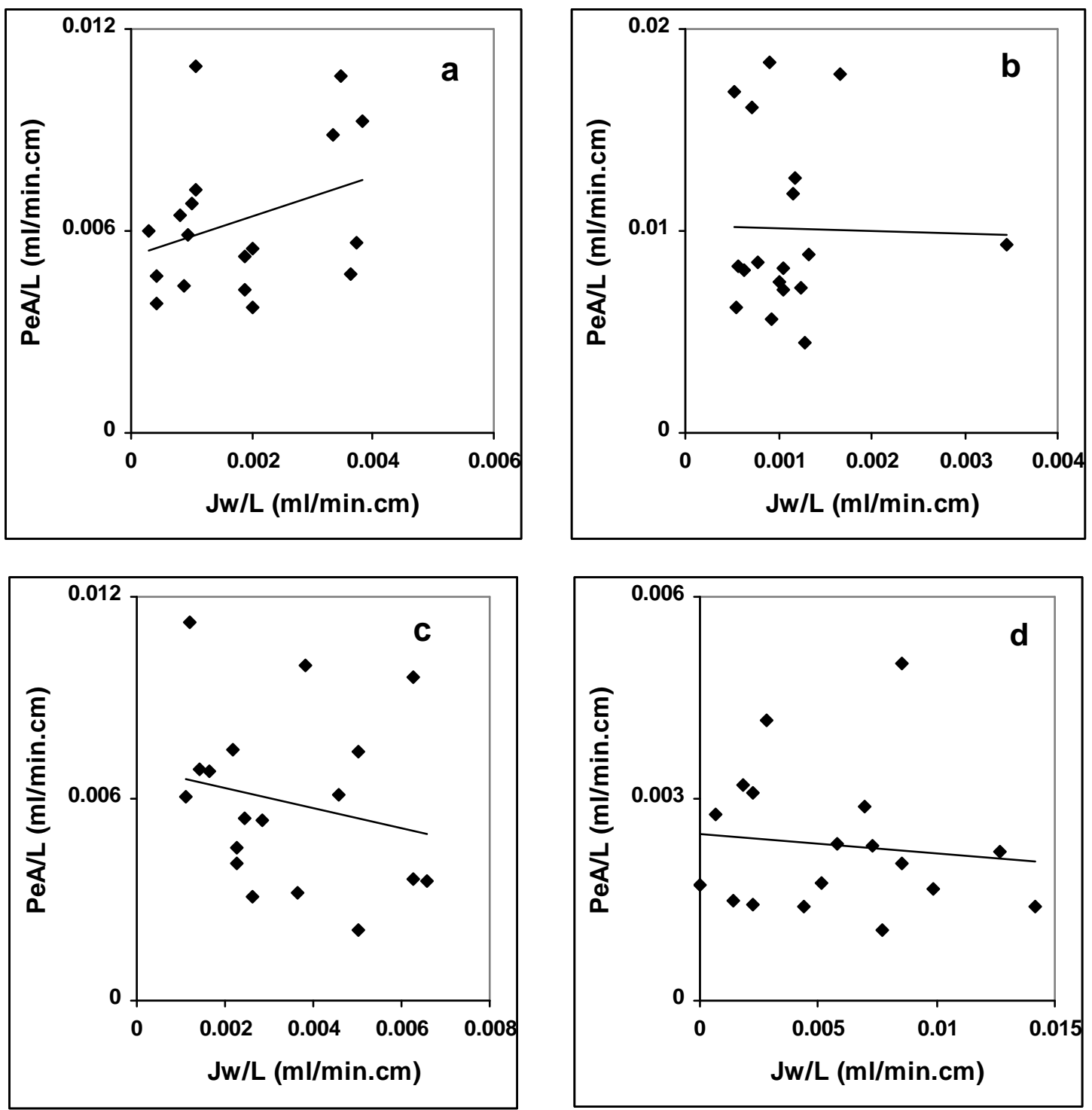

Fig. 2: Absorptive clearance of sildenafil versus water flux in different intestinal segments; (a) duodenum, (b) Jejunoileum, (c) colon and (d) rectum. Parameters are normalized to segment length. 
Table 2: Effect of water flux $(\mathrm{ml} / \mathrm{min} . \mathrm{cm})$ on the overall absorptive clearance (ml/min.cm), of sildenafil. Regression parameters are obtained by fitting data to equation 8 .

\begin{tabular}{|c|c|c|}
\hline Segment & $\begin{array}{c}\text { Intercept } \\
(\mathrm{DAKp} / \Delta \mathrm{x})\end{array}$ & Slope $(\varnothing \mathrm{s})$ \\
\hline Duodenum & $\begin{array}{c}0.00526 * * * \\
(0.00091)\end{array}$ & $\begin{array}{c}0.5857^{*} \\
(0.4173)\end{array}$ \\
\hline Jejunoileum & $\begin{array}{c}0.0103 * * * \\
(0.0021)\end{array}$ & $\begin{array}{c}-0.128^{*} \\
(1.663)\end{array}$ \\
\hline Ascending & $\begin{array}{c}0.00694 * * * \\
(0.00131)\end{array}$ & $\begin{array}{c}-0.301 * \\
(0.343)\end{array}$ \\
\hline colon & $\begin{array}{c}0.00249 * * * \\
\text { Rectum }\end{array}$ & $-0.0291^{*}$ \\
$(0.000440)$ & $(0.0633)$ \\
\hline
\end{tabular}

Values in parentheses are the standard error values, $n=3$. $(D A K p / \Delta x)$ is the permeability coefficient and $(\varnothing \mathrm{s})$ is the sieving coefficient. *Not significantly different from zero $(\mathrm{P}>0.05)$. $* * *$ Significantly different from zero $(\mathrm{P}<0.01)$.

The plasma sildenafil concentration achieved at the end of the intestinal perfusion experiment was calculated from the average value of the last three concentrations. The last three concentrations were always within $10 \%$ indicating that these concentrations can be a good approximation of the steady state concentration. The bioavailability of sildenafil during the intestinal perfusion experiment was calculated in each rabbit from the rate of drug disappearance from the perfused segment, the plasma concentration and the average estimate for sildenafil total body clearance. The calculated parameters for sildenafil presystemic elimination during the intestinal perfusion experiment are summarized in Table 3.

The oral bioavailability of sildenafil in human has been reported to be $38-41 \% .^{3-5}$ It is important to investigate the cause of this incomplete bioavailability in order to determine the factors that may alter the drug bioavailability and hence its pharmacological effects. Also, proper design of dosage form in order to optimize drug absorption and bioavailability depends on understanding the membrane transport characteristics and the mechanisms of drug absorption.

The absorption of sildenafil is different in the four different GIT segments investigated in the current study with the highest absorption occurring from the jejunoileum followed by the duodenum, the ascending colon and the rectum, respectively. This was apparent from the different fraction of the drug absorbed during the perfusion experiments, the absorptive clearance normalized for the segment length, and the anatomical reserve length (Table 1). These results may suggest that the absorption of sildenafil occurs mainly from the jejunoileum and that slowing the gastric emptying rate may lead to slowing sildenafil absorption. It should be noted that the length of jejunoileum required for $95 \%$ absorption was only $87.58 \mathrm{~cm}$. this indicates that the oral absorption of sildenafil is nearly complete.

Table 3: Sildenafil pharmacokinetic parameter and presystemic elimination parameters.

\begin{tabular}{|l|c|c|c|c|c|}
\hline \multicolumn{1}{|c|}{ Parameters } & $\begin{array}{c}\text { Rabbit } \\
1\end{array}$ & $\begin{array}{c}\text { Rabbit } \\
2\end{array}$ & $\begin{array}{c}\text { Rabbit } \\
3\end{array}$ & Mean & SD \\
\hline Sildenafil perfusion rate $\left(\mathrm{R}_{\text {in }}, \mu \mathrm{g} / \mathrm{min}\right)$ & \multicolumn{5}{|c|}{3.672} \\
\hline $\begin{array}{l}\text { Amount of sildenafil disappeared during } \\
\text { the intestinal perfusion }\left(\mathrm{R}_{\text {in }}-\mathrm{R}_{\text {out }}, \mu \mathrm{g} / \mathrm{min}\right)\end{array}$ & 3.345 & 3.275 & 3.460 & 3.360 & 0.093 \\
\hline $\begin{array}{l}\text { Average plasma concentration achieved } \\
\text { during the intestinal perfusion } \\
\text { experiment. }(\mu \mathrm{g} / \mathrm{ml})\end{array}$ & 0.0514 & 0.0987 & 0.0453 & 0.0651 & 0.029 \\
\hline $\begin{array}{l}\text { Fraction of sildenafil that reaches the } \\
\text { systemic circulation during the intestinal } \\
\text { perfusion* }\end{array}$ & 0.27 & 0.53 & 0.23 & 0.343 & 0.163 \\
\hline Pre-Systemic elimination & $73 \%$ & $47 \%$ & $77 \%$ & 65.7 & 16.3 \\
\hline
\end{tabular}

*This was calculated from equation 9 , taking the average value of $\mathrm{CL}_{\text {tot }}$ as $17.57 \mathrm{ml} / \mathrm{min}$. 
The poor correlation between the absorptive clearance of sildenafil and the net water flux in the different GIT segments investigated in this study indicates that its absorption is primarily though transcellular pathway. This is apparent from the slope and the intercept of the regression between the absorptive clearance and the net water flux. This can explain the different sildenafil absorption from the different GIT segments, which have different surface area per unit length. The extent of sildenafil absorption from the different anatomical segments is in the same order of the surface area per unit length with jejunoileum showing the highest and the rectum revealing the lowest absorption.

The results of this investigation clearly indicated complete absorption of sildenafil from the GIT. This excludes any role for the membrane transport in the reported poor bioavailability of sildenafil. Accordingly, the presystemic disposition of sildenafil was considered. Our investigation showed that about $66 \%$ of the absorbed drug was eliminated before reaching the systemic circulation. This presystemic elimination resulted in only $34 \%$ of the absorbed drug to be bioavailable. The primary Cytochrome $\mathrm{P} 450$ isoform responsible for sildenafil metabolism is mainly CYP3A4, with CYP2C9 playing a minor role. ${ }^{21}$ These CYP450 isoforms are present in large amounts in the intestine and the liver and are responsible for the extensive presystemic elimination of sildenafil. The clinical significance of this is that administration of sildenafil with inhibitors of these CYP450 isoforms such as cimetidine, macrolide antibiotics and protease inhibitors, can significantly increase sildenafil bioavailability which can lead to augmentation of its pharmacological and toxicological effects. ${ }^{22-23}$

The results indicated that improved sildenafil bioavailability after oral administration cannot be achieved by enhancing drug absorption because its absorption is almost complete from the GIT. The improved bioavailability can be obtained by avoiding the presystemic metabolism. This can be achieved for example through the rectal route. However, our study revealed poor rectal absorption of sildenafil. Thus if rectal dosage form is to be designed an absorption enhancer must be included. The results of our study suggested mainly a transcellular absorption of the drug from all segments. Thus the absorption enhancer must be selected among those acting on the transcellular mechanism (such as bile salts) and those influencing the paracellular route must be excluded.

\section{Conclusions}

The intestinal absorption of sildenafil citrate was site dependent with the absorption in the order of jejunoileum > duodenum > ascending colon $>$ rectum. The study revealed apparently complete absorption from GIT with the absorption being mainly transcellular and no evidence for paracellular mechanism in all segments.

Presystemic elimination was mainly responsible for poor bioavailability. Accordingly, avoidance of the presystemic metabolism is the recomended approach to optimize sildenafil bioavailability. Finally, if rectal dosage form is to be designed, an absorption enhancer must be included. This enhancer must improve transcellular absorption which is the major pathway for sildenafil permeation.

\section{REFERENCES}

1- D. Vitezic and J. M. Pelcic, Int. J. Clin. Pharmacol. Ther., 40, 393 (2002).

2- C. C. Carson, Expert Opin. Pharmacother. 4, 397 (2003).

3- G. J. Muirhead, D. J. Rance, D. K. Walker and P. Wastall, Br. J. Clin. Pharmacol. 53, 13S (2002).

4- D. J. Nichols, G. J. Muirhead and J. A. Harness, Br. J. Clin. Pharmacol. 53, 5S(2002).

5- M. De Siati, M. Saugo, and N. Franzolin, Arch. Ital. Urol. Androl., 75, 18 (2003).

6- S. A. Kaplan and M. L. Jack, In Vitro, In Situ, and In Vivo Models In Bioavailability Assessment. In: Blanchard, J., Sawchuk, R.J., Brodie, B. B., Ed., Principles and Perspectives In Drug Bioavailability, S. Karger, Basel, Switzerland, 1979; pp.15691.

7- L. E. Riad and R. J. Sawchuk, Pharm. Res., 8, 1050 (1991).

8- J. P. Zhong, Y. F. Wang and R. J. Sawchuk, Pharm. Res., 9, S350 (1992). 
9- M. A. Osman, M. K. Youssef and R. J. Sawchuk, Alexandria Second International Conference of Pharmaceutical Sciences and Technology 2000, Abstract Book, p. 56.

10- C. A. Loehry, J. Kingham and J. Baker, Gut, 141, 683 (1973).

11- T. Maeda, H. Takenaka, Y. Yamahira and T. Noguchi, J. Pharm. Sci., 66, 69 (1977).

12- D. N. Granger, P. R. Kvietys, M. A. Perry and J. A. Barrowman, The Microcirculation And Intestinal Transport. In: Johnson, L. R., Physiology of the Gastrointestinal Tract, $2^{\text {nd }}$ Ed., Raven Press, NY 1987, Vol. 2, pp. 1671-97.

13- G. E. Amidon, N. F. H. Ho, A. B. French and W. I. Higushi, J. Theo. Biol., 89, 195 (1981).

14- I. Komiya, J. Y. Park, A. Kamani, N. F. H. Ho and W. I. Higuchi, Int. J. Pharm., 4, 249 (1980).

15- P. F. Ni, N. F. H. Ho, J. L. Fox, H. Leuenberger and W. I. Higuchi, Ibid, 5, 33 (1980).
16- N. F. H. Ho, H. P. Merkle, and W. I. Higuchi, Drug Dev. Ind. Pharm., 9, 1111 (1983).

17- G. Gan, L. L. Cartier, Y. Huang, Z. Yang and R. J. Sawchuk, J. Pharm. Sci., 91, 217 (2002).

18- N. Lifson and A. A. Hakim, Urea. Am. J. Physiol., 211, 1137 (1966).

19- N. Lifson, L. M. Gruman and D. G. Levitt, Ibid, 215,444 (1968).

20 - E. H. Bresler and L. J. Groome, Ibid , 241, F 469 (1981).

21- J. S. Warrington, R. I. Shager, L. I. Von Molte and D. J. Green blatt, Drug Metab. Dispos., 28, 392 (2000).

22- K. Wilner, L. Labey and M. Lersel, Br. J. Clin Pharmacol., 53, 13s (2002).

23- G. J. Muirhead, S. Faulken, J. A. Harness and J. Taubel, Br J Clin Pharmacol., 53, 37s (2002). 